\title{
Peer victimization and the association with hippocampal development and working memory in children with and without ADHD
}

\author{
Alissa Papadopoulos \\ Western University \\ Diane Seguin \\ Western University
}

\section{Susana Correa}

Western University

Emma G. Duerden ( $\square$ eduerden@uwo.ca )

Western University

\section{Research Article}

Keywords: ADHD, executive functioning, externalizing behaviours, hippocampus, peer victimization, working memory, child and youth mental health

Posted Date: April 9th, 2021

DOl: https://doi.org/10.21203/rs.3.rs-402441/v1

License: (a) (i) This work is licensed under a Creative Commons Attribution 4.0 International License. Read Full License

Version of Record: A version of this preprint was published at Scientific Reports on August 12th, 2021. See the published version at https://doi.org/10.1038/s41598-021-95582-7. 


\section{Abstract \\ Background}

Children with ADHD are at risk of experiencing peer victimization, which is associated with delayed brain development and cognitive difficulties. We aimed to evaluate the relationship between problem behaviours, peer victimization, hippocampal morphology, and working memory in children with and without ADHD.

\section{Methods}

218 typically developing participants (50.5\% male) and 232 participants diagnosed with ADHD (77.6\% male) were recruited. The ADHD group was subdivided into inattentive (ADHD-I) or combined (ADHD-C) type. The Child Behaviour Checklist measured problem behaviours and peer victimization. Hippocampal subfield volumes were obtained using Magnetic Resonance Imaging and the Wechsler Intelligence Scale for Children-fifth edition measured working memory (WM).

\section{Results}

The ADHD-C group displayed significantly higher rates of problem behaviours and peer victimization (all, $\mathrm{p}<0.001$ ). Left Cornu Ammonis 3 (CA3) volume was a positive predictor of levels of peer victimization (all, $p<0.013$ ). Left CA3 volume was a positive predictor of WM and left Cornu Ammonis 4 (CA4) volume was a negative predictor (all, $p<0.025$ ). A cluster analysis revealed that children displaying symptoms of hyperactivity-impulsivity are the most at risk for peer victimization.

\section{Conclusions}

Interventions focusing on minimizing peer victimization may aid in mitigating adverse downstream effects, and aid in promoting brain health and cognitive function.

\section{Introduction}

Attention Deficit Hyperactivity Disorder (ADHD) affects approximately $5 \%$ of the child and youth population worldwide and is characterized by symptoms of hyperactivity-impulsivity, and inattention ${ }^{1,2}$. $A D H D$ is further characterized into three diagnostic subtypes, including ADHD hyperactive-impulsive type (ADHD-H), ADHD inattentive type (ADHD-I), and ADHD combined type (ADHD-C) ${ }^{1,3}$. ADHD-H is characterized by clinically elevated symptoms of hyperactivity-impulsivity ${ }^{1}$. ADHD-I is characterized by clinically elevated symptoms of inattention. Children and youth with ADHD-C display both clinically elevated hyperactivity-impulsivity and inattention symptoms ${ }^{1}$. 
Children with ADHD have deficits in social skills and often act inappropriately in social situations, such as being disruptive and intrusive, which make maintaining healthy peer relationships difficult ${ }^{4,5}$. These social difficulties make children with ADHD susceptible to peer victimization and research has suggested that children with ADHD experience the highest rates of peer victimization when compared to children with other disabilities such as Autism Spectrum Disorder (ASD), Oppositional Defiant Disorder (ODD), intellectual disabilities, learning disabilities, and physical disabilities ${ }^{4,5,6}$. However, recent research has demonstrated that the levels of peer victimization experienced by children with ADHD vary according to their diagnosis subtype, with symptoms of hyperactivity-impulsivity centrally implicated as a factor that increases risk of experiencing higher levels of peer victimization ${ }^{7}$.Children with more severe hyperactivityimpulsivity symptoms also tend to exhibit more problem behaviours, such as rule breaking and externalized aggression, which can negatively influence the ability to form healthy peer relationships ${ }^{4,5}$. This suggests that children and youth with ADHD-H and ADHD-C are more susceptible to experiencing peer victimization than those with ADHD-I. Peer relationships play an important role in child development, and victimization by peers, including verbal, emotional, and physical abuse, can be a significant and potentially traumatizing stressor in a young person's life $e^{8,9}$.

Children with ADHD differ in their brain structure compared to their TD counterparts ${ }^{10}$. However, for many brain regions, including the hippocampus, how the structure differs between ADHD and TD children is unclear $^{11,12,13,14,15}$. In some studies, enlarged hippocampal volumes are reported in ADHD children compared to TD children ${ }^{12}$, some studies report decreased hippocampal volumes in ADHD compared to TD children ${ }^{13,14}$, and others report no differences ${ }^{15}$.

The hippocampus is a structure of particular interest in the adversity literature because it is involved in regulating hormonal stress responses and is composed of stress-sensitive subfields including the Cornu Ammonis 1 (CA1), Cornu Ammonis 3 (CA3), and Cornu Ammonis 4 (CA4) ${ }^{16,17}$. It is also of particular interest of study in the ADHD population, considering children with ADHD are at an increased risk of experiencing chronic stress, including that related to peer victimization $12,13,14,15$.

Chronic stress has been hypothesized to impact the hippocampus in different ways. For one, during periods of increased stress, the adrenal gland releases glucocorticoids (GCs) ${ }^{17}$. In rodent and primate models, GCs have been observed to kill neurons in stress-sensitive subfields of the hippocampus, for example in the CA3, thereby leading to decreased volumes ${ }^{17}$. Other research suggests that chronically elevated levels of stress might lead to a reduced hormonal stress responsivity that can mitigate the neurotoxic effects of GCs, resulting in unaltered or even enlarged hippocampal volumes ${ }^{17,18,19}$. In the literature, both decreased and enlarged hippocampal volumes have been observed. Some studies have demonstrated that early life stress is associated with decreased hippocampal volumes, although in some pediatric populations, such as in children with maltreatment-related PTSD, larger hippocampal volumes have been observed in relation to increased adversity ${ }^{16,20,21}$. 
The research on peer victimization as a form of early life stress and its association with hippocampal volume is scarce. In some studies, smaller hippocampal volumes were found in TD children who reported more peer victimization, but further research is required to obtain a better understanding of this relationship ${ }^{9,22}$. Further, the association between peer victimization to hippocampal volume in the ADHD population and how this differs between ADHD subtypes has not been studied.

In addition to its involvement in regulating the stress response, the hippocampus is also a key structure in executive functioning ${ }^{23}$. In adult and aging populations, larger hippocampal volumes are associated with better performance on tasks of executive functioning, including working memory (WM) $23,24,25$. However, this same relationship is not consistently observed in children, with some evidence suggesting that children with larger hippocampal volumes perform worse on tasks of memory ${ }^{24}$. Children with ADHD exhibit deficits in WM, however the relationship between hippocampal volume and WM ability in the ADHD population remains largely unexplored ${ }^{26}$.

In the current work, we examined the association amongst social difficulties, brain development and executive function in a large heterogenous sample of children with ADHD and TD children. We addressed three main research questions: 1) Are problem behaviours and peer victimization predicted by ADHD diagnostic category? 2) Do hippocampal subfield volumes predict problem behaviours and peer victimization levels? 3) Do hippocampal subfield volumes predict WM ability? Our central hypothesis is that ADHD participants will report higher levels of problem behaviours and peer victimization compared to TD children, and that hippocampal subregion volumes will significantly predict levels of peer victimization as well as WM ability.

\section{Methods}

\section{Participants}

Participants in this study ranged in age from 6.00 to 17.70 years. Data were collected by researchers at the Child Mind Institute and participants were recruited as part of the Healthy Brain Network initiative ${ }^{27}$. Both typically developing (TD) participants and those with a clinical diagnosis of ADHD were tested at three sites: Rutgers University, New York, Citigroup Biomedical Imaging Center, Weill Cornell Medicine, New York, and Staten Island Diagnostic Research Center, New York. The research ethics boards at all respective institutions approved the study. Written informed consent was obtained from the participants' parents and written assent was obtained from the participants. A breakdown of participant demographics can be found in Table 1. 
Table 1

Characteristics of TD, ADHD-C and ADHD-I participants.

\begin{tabular}{|c|c|c|c|}
\hline & \multirow{2}{*}{$\begin{array}{l}\text { TD } \\
\mathrm{n}=\mathbf{2 1 8}\end{array}$} & \multirow{2}{*}{$\begin{array}{l}\text { ADHD-C } \\
n=108\end{array}$} & \multirow{2}{*}{$\begin{array}{l}\text { ADHD-I } \\
n=124\end{array}$} \\
\hline & & & \\
\hline Age, years, & 9.80 & 8.80 & 10.60 \\
\hline Median [IQR] & [7.90-12.72] & [7.26-11.24] & [8.58-13.05] \\
\hline Male, \% (n) & $50.5(110)$ & $79.6(86)$ & $75.8(94)$ \\
\hline MRI Site, \% (n)† & $14.2(31)$ & $25.0(27)$ & $27.0(34)$ \\
\hline CBIC & $34.9(76)$ & $31.5(34)$ & $47.6(59)$ \\
\hline $\mathrm{RU}$ & $26.1(57)$ & $16.7(18)$ & $5.60(7)$ \\
\hline SI & $0.9(2)$ & $0(0)$ & $0(0)$ \\
\hline Unknown & 53.00 & 53.00 & 53.00 \\
\hline BSMSS Total, & [46.00-61.00] & [44.88-61.00] & [45.00-59.50] \\
\hline Median [IQR] & $-0.11[-1.42-0.22]$ & 1.11 [0.67-1.67] & $0.22[0.00-0.67]$ \\
\hline SWAN, Median [IQR] & $-0.06[-1.22-0.44]$ & 1.11 [0.67-1.67] & $1.22[0.56-1.89]$ \\
\hline \multirow{2}{*}{$\begin{array}{l}\text { Hyperactivity- } \\
\text { Impulsivity }\end{array}$} & $3.00[0.00-5.00]$ & $8.00[4.25-13.00]$ & $4.00[1.00-8.00]$ \\
\hline & $1.00[0.00-2.00]$ & $3.00[1.00-5.00]$ & $2.00[0.00-3.00]$ \\
\hline \multirow{2}{*}{$\begin{array}{l}\text { Inattention } \\
\text { CBCL, Median [IQR] }\end{array}$} & $1.00[0.00-3.00]$ & $3.50[1.00-6.00]$ & $2.00[0.00-3.75]$ \\
\hline & $1.00[0.00-2.00]$ & $1.00[0.00-3.00]$ & $1.00[0.00-3.00]$ \\
\hline Aggressive Behaviour & $0.00[0.00-0.00]$ & $1.00[0.00-2.00]$ & $0.00[0.00-1.00]$ \\
\hline $\begin{array}{l}\text { Rule Breaking } \\
\text { Behaviour }\end{array}$ & 106.00 & 105.00 & 99.00 \\
\hline Social Problems & [95.00-113.00] & [91.75-113.00] & [88.00-109.00] \\
\hline Withdrawn & 100.00 & 100.00 & 94.00 \\
\hline Peer Victimization & [91.00-112.00] & [91.00-110.00] & [88.00-107.00] \\
\hline WISC-V, Median [IQR] & 1176718.50 & 1202310.00 & 1217219.00 \\
\hline FSIQ & $\begin{array}{l}{[1104748.00-} \\
1268853.75]\end{array}$ & $\begin{array}{l}{[1104571.00-} \\
1260378.00]\end{array}$ & $\begin{array}{l}{[1142320.00-} \\
1334728.00]\end{array}$ \\
\hline WMI & 178.68 & 178.74 & 200.11 \\
\hline \multicolumn{4}{|c|}{ 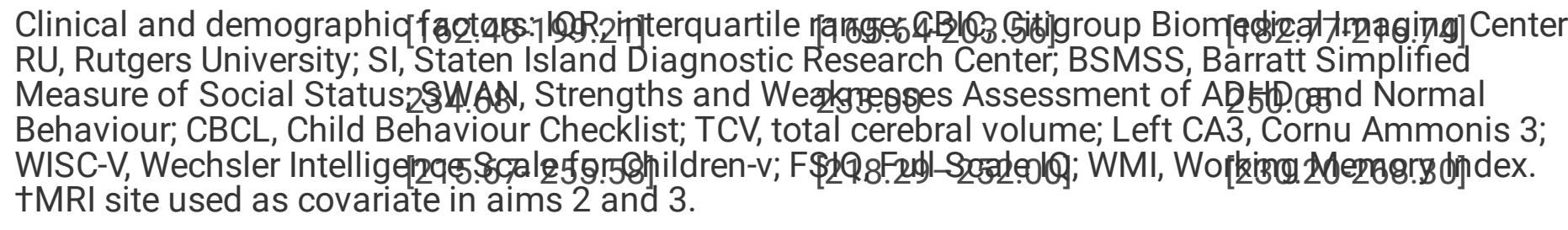 } \\
\hline
\end{tabular}




\begin{tabular}{|c|c|c|c|}
\hline & $\begin{array}{l}\text { TD } \\
\mathrm{n}=\mathbf{2 1 8}\end{array}$ & $\begin{array}{l}\text { ADHD-C } \\
n=108\end{array}$ & $\begin{array}{l}\text { ADHD-I } \\
n=124\end{array}$ \\
\hline \multicolumn{4}{|l|}{$\mathrm{TCV}\left(\mathrm{mm}^{3}\right)$} \\
\hline \multicolumn{4}{|l|}{ Median [IQR] } \\
\hline \multicolumn{4}{|l|}{ Left CA3 $\left(\mathrm{mm}^{3}\right)$, } \\
\hline \multicolumn{4}{|l|}{ Median [IQR] } \\
\hline \multicolumn{4}{|l|}{ Left CA4 $\left(\mathrm{mm}^{3}\right)$} \\
\hline \multicolumn{4}{|l|}{ Median [IQR] } \\
\hline \multicolumn{4}{|c|}{$\begin{array}{l}\text { Clinical and demographic factors: IQR, interquartile range; CBIC, Citigroup Biomedical Imaging Center; } \\
\text { RU, Rutgers University; SI, Staten Island Diagnostic Research Center; BSMSS, Barratt Simplified } \\
\text { Measure of Social Status; SWAN, Strengths and Weaknesses Assessment of ADHD and Normal } \\
\text { Behaviour; CBCL, Child Behaviour Checklist; TCV, total cerebral volume; Left CA3, Cornu Ammonis 3; } \\
\text { WISC-V, Wechsler Intelligence Scale for Children-V; FSIQ, Full Scale IQ; WMI, Working Memory Index. } \\
\text { TMRI site used as covariate in aims } 2 \text { and } 3 \text {. }\end{array}$} \\
\hline
\end{tabular}

In combination with clinical judgment, the computerized version of the Kiddie Schedule for Affective Disorders and Schizophrenia (K-SADS-COMP) was administered under the supervision of a licensed clinician to make diagnoses of ADHD, and to confirm the absence of ADHD diagnoses in TD participants ${ }^{28}$. Participants diagnosed with ADHD were further divided into subgroups, those diagnosed with ADHD-I and those diagnosed with ADHD-C. Participants with an ADHD-H diagnosed were excluded from this study due to a small sample size of only 15 participants having received this subtype diagnosis. All participants in the study were fluent in English, had a parent that was able to complete informant questionnaires, had an Intelligence Quotient (IQ) score of over 66, and were free of brain injury or disease. Participants were excluded if they presented with schizophrenia or bipolar disorder, psychosis, substance dependence, acute intoxication, a neurodegenerative disorder, or any other neurodevelopmental disorder. The study was conducted in accordance with the Declaration of Helsinki.

\section{Procedures}

The data for this study was collected in 4 study visits. In the first visit, the WISC-V was administered by a trained psychometrist. The second visit consisted of the MRI protocol, including the T1 sequence conducted by a trained MRI technician. During the scan participants watched two cartoon movies, the first movie was a short-film titled 'The Present' and the second was a 10-minute clip from the full-length film 'Despicable Me.' Parent questionnaires were completed during the third visit and K-SADS-COMP was completed during the fourth and final visit.

\section{Demographic Measures}


The Barratt Simplified Measure of Social Status (BSMSS) was used as a measure of socioeconomic status (SES) and was completed by a parent (Barratt, 2006). The BSMSS assesses level of education and occupation for both of a child's parents, or from one parent if the child is from a single-parent household ${ }^{29}$. The scores were converted into a total score between 8-66 for each child, with a higher score indicative of a higher SES $^{29}$.

\section{Psychological Measures}

To assess symptoms of hyperactivity-impulsivity and inattention, the Strengths and Weaknesses Assessment of ADHD and Normal Behaviour (SWAN) was completed by a parent (Swanson et al., 2001). To measure levels of problem behaviours in the participants, the Child Behaviour Checklist (CBCL) was administered to parents ${ }^{30}$. The subscales used from the CBCL in this study included the aggressive behaviour, rule breaking behaviour, social problems and withdrawn scales. In addition, peer victimization was measured using the CBCL subscale developed by McCloskey and Stuewig ${ }^{31}$, comprised of four questions: "doesn't get along with other kids", "gets in many fights", "gets teased a lot", and "not liked by other children," The Wechsler Intelligence Scale for Children-fifth edition (WISC-V) was used to obtain a Working Memory Index (WMI) in all participants, including a digit span task and a picture span task ${ }^{32}$.

\section{Magnetic Resonance Imaging}

A Siemens $3 T$ Trio scanner was used at the Rutgers University site ${ }^{27}$. At the Staten Island Diagnostic Research Center, participants were scanned on a 1.5T Siemens Avanto scanner and at Citigroup Biomedical Imaging Center participants were scanned on a Siemens 3T Prisma ${ }^{27}$. To adjust for the differences in scanners, statistical models included Magnetic Resonance Imaging (MRI) site as a covariate.

High-resolution anatomical images were acquired using a 3D-MPRAGE pulse sequence with 192 T1weighted, straight sagittal slices (1 mm thickness).

\section{Hippocampal Segmentation}

To obtain hippocampal subfield volumes from the structural images, FreeSurfer (http://surfer.nmr.mgh.harvard.edu) version 6.0. was used to segment and isolate hippocampal structures at high-resolution (Iglesias et al., 2015). The hippocampus was segmented, bilaterally, into the hippocampal tail, subiculum, CA1, hippocampal fissure, presubiculum, parasubiculum, molecular layer, granule cell layers of the dentate gyrus (GCMLDG), CA3, CA4, fimbria, and the hippocampus-amygdalatransition-area (HATA). The total cerebral volumes were extracted using the Freesurfer pipeline.

\section{Statistical Analysis}

Statistical analyses were completed the IBM SPSS Statistics software package (version 26, Statistical Package for the Social Sciences, IBM, Armonk, NY). 
Our first aim was to examine problem behaviour and peer victimization in children and adolescents with TD, ADHD-I, ADHD-C. In five general linear models, we examined parent-reported problem behaviours and peer victimization (social problems, aggressive behaviour, rule breaking behaviour, withdrawal and peer victimization subscale raw scores; dependent variables) in related to diagnostic group (TD, ADHD-I, ADHD-C; independent variables). Age, sex, socioeconomic status (using the BSMSS), and study site/MRI site were adjusted for in each model.

Our second aim was to examine problem behaviour and peer victimization and their relationships to hippocampal subfield volume. In four general linear models we examined peer victimization (subscale raw score; dependent variable), aggressive behaviour (subscale raw score; dependent variable), rule breaking behaviour (subscale raw score; dependent variable), and social problems (subscale raw score; dependent variable) in relation to hippocampal subfield volumes (right and left hippocampal tail, subiculum, CA1, hippocampal fissure, presubiculum, parasubiculum, molecular layer, GCMLDG, CA3, CA4, fimbria and HATA; independent variables), while adjusting for age, sex, socioeconomic status (using the BSMSS), diagnostic group, total cerebral volume (TCV), and MRI site.

Our third aim was to examine the association of hippocampal subfield volumes and working memory ability. A general linear model was used to examine the relationship between the left and right hippocampal subfield volumes (hippocampal tail, subiculum, CA1, hippocampal fissure, presubiculum, parasubiculum, molecular layer, GCMLDG, CA3, CA4, fimbria and HATA; independent variables), and working memory ability (WISC-V: working memory subscale standardized score; dependent variable). We adjusted for age, sex, socioeconomic status (using the BSMSS), diagnostic group, TCV, and MRI site.

\section{Results}

\section{Participant Demographics}

A total of 218 TD participants (median age $=9.80$ years; $50.5 \%$ male) and 232 participants diagnosed with ADHD (median age $=9.82$ years; $77.6 \%$ male) were recruited. Of the 232 children and adolescents diagnosed with ADHD, 108 participants had the ADHD-C subtype (median age $=8.80$ years; interquartile range $[\mathrm{IQR}]=7.26-11.24$ years; $79.6 \%$ male) and 124 participants had the ADHD-I subtype (median age $=$ 10.60 years; IQR $=8.58-13.05$ years; $75.8 \%$ male). Participant characteristics can be found in Table 1 .

\section{Problem Behaviours, Peer Victimization and Diagnostic Group}

In our first aim, we examined the relationship between problem behaviours from the $\mathrm{CBCL}$, including social problems, rule breaking behaviour and aggressive behaviour, and diagnostic group (TD, ADHD-I, and ADHD-C). We also examined how the experience of peer victimization differs by diagnostic group (TD, ADHD-I, and ADHD-C). Age, sex, SES, and study site were adjusted for in all the models. In the problem behaviours models 433 participants were included in analysis, 204 TD children, 123 ADHD-I children, and 106 ADHD-C children. 
Seventeen participants were excluded from the problem behaviour analyses due to missing data. In the peer victimization model a total of 431 total participants were included in the analysis, 203 TD children, 123 ADHD-I children, and 105 ADHD-C children.

Compared to the TD group, children and adolescents from both the ADHD-C $(B=5.42,95 \% \mathrm{Cl}=4.26-6.58$, $p<0.001)$ and $A D H D-I(B=2.41,95 \% C l=1.32-3.51, p<0.001)$ groups had significantly higher scores on the aggressive behaviour subscale. ADHD-C participants also had significantly higher scores than the TD participants on the rule breaking behaviour $(B=1.95,95 \% \mathrm{Cl}=1.39-2.50, p<0.001)$ and social problems $(B=2.16,95 \% \mathrm{Cl}=1.53-2.78, p<0.001)$ subscales. No significant differences were found between the TD and ADHD-C participants on the withdrawn $(B=0.51,95 \% \mathrm{Cl}=0.03-0.99, \mathrm{p}=0.038)$ subscale. Nor were significant differences found between the TD and $A D H D-I$ participants on the rule breaking behaviour $(B=$ $0.62,95 \% \mathrm{Cl}=0.10-1.15, \mathrm{p}=0.021)$, social problems $(\mathrm{B}=0.75,95 \% \mathrm{Cl}=0.16-1.33, \mathrm{p}=0.013)$, or withdrawn $(B=0.49,95 \% \mathrm{Cl}=.04-0.94, p=0.033)$ subscales. In comparing ADHD-C to ADHD-I children, we found that the ADHD-C children had significantly higher scores on aggressive behaviour, rule breaking behaviour, and social problems (all, $\mathrm{p}<0.001$ ).

When examining the peer victimization subscale, children with ADHD-C had significantly higher levels of peer victimization compared TD children $(B=0.73,95 \% \mathrm{Cl}=0.47-0.98, p<0.001)$, but there was no significant difference found for peer victimization between the ADHD-I and TD groups $(B=0.09$, $95 \% \mathrm{Cl}=-0.16-0.33, \mathrm{p}=0.483)$. In addition, the ADHD-C children had significantly higher peer victimization scores than the ADHD-I children ( $p$ 0.001). Refer to Fig. 1 for a summary of the results.

\section{Problem Behaviours, Peer Victimization and Hippocampal Subfield Volumes}

In our second aim, we examined whether hippocampal subfield volumes could predict problem behaviours and levels of peer victimization. The problem behaviours examined in this aim included aggressive behaviour, rule breaking behaviour, and social problems subscales, adjusting for age, sex, SES, MRI site, diagnostic group, and TCV. The analysis was run with a total of 282 participants, including 136 TD children, 83 ADHD-I children, and 63 ADHD-C children.

Left CA3 volume was positively associated with peer victimization $(B=0.019,95 \% \mathrm{Cl}=0.005-0.034, p=$ 0.010). Left $C A 3$ volume showed a positive but not-significant association with rule breaking behaviour $(B=0.034,95 \% \mathrm{Cl}=0.003-0.066, p=0.032)$, aggressive behaviour $(B=0.054,95 \% C l=-0.010-0.119, p=$ 0.097), and social problems $(B=0.029,95 \% \mathrm{Cl}=-0.006-0.064, \mathrm{p}=0.107)$.

We subsequently examined the interaction between left CA3 volume and diagnostic group (left CA3 volume $x$ diagnostic group) in the peer victimization model. We found that left CA3 volume was significantly and positively associated with peer victimization in in TD children $(B=0.018,95 \% \mathrm{Cl}=0.003-$ $0.33, p=0.017)$, ADHD-C children $(B=0.022,95 \% C l=0.007-0.036, p=0.004)$, and $A D H D-I$ children $(B=$ $0.017,95 \% \mathrm{Cl}=0.003-0.032, \mathrm{p}=0.022)$. 


\section{Hippocampal Subfield Volumes and Working Memory Ability}

This analysis included a total of 243 participants, including 109 TD children, 76 ADHD-I children, and 58 ADHD-C children. When adjusting for age, sex, SES, MRI site, TCV, and hippocampal subfield volumes the ADHD-I group had significantly poorer WM scores than the TD group $(B=-5.49,95 \% \mathrm{Cl}=-9.75--1.23, p=$ $0.011)$, but the ADHD-C group did not significantly differ from the TD group $(B=-4.31,95 \% C l=-8.95-0.33$, $p=0.069)$.

We examined the relationship between hippocampal subfield volumes and WMI (WISC-V). Left CA3 volume was found to be significantly and positively associated with $\mathrm{WMI}(\mathrm{B}=0.233,95 \% \mathrm{Cl}=0.042-$ $0.424, p=0.017)$. Statistical interactions were tested in the WMI model between left CA3 volume and diagnostic group (left CA3 $x$ diagnostic group). Left CA3 volume was significantly and positively associated with WMI in TD children $(B=0.249,95 \% \mathrm{Cl}=0.59-0.440, p=0.010)$, ADHD-C children $(B=$ $0.230,95 \% \mathrm{Cl}=0.038-0.422, \mathrm{p}=0.019)$, and $A D H D-I$ children $(B=0.220,95 \% \mathrm{Cl}=0.30-0.411, p=0.023)$.

Left CA4 volume was found to be significantly and negatively associated with WMI $(B=-0.626$, $95 \% \mathrm{Cl}=-1.14--0.107, p=0.018)$. Statistical interactions were also tested in the WMI model between left CA4 and diagnostic group. Left CA4 volume was significantly and negatively associated with WM in TD children $(B=-0.608,95 \% C l=-1.128--0.089, p=0.022)$, ADHD-C children $(B=-0.625,95 \% C l=-1.141--0.108, p$ $=0.018)$, and $A D H D-I$ children $(B=-0.632,95 \% C l=-1.151--0.113, p=0.017)$.

\section{Post-Hoc Cluster Analysis}

To examine the associations amongst diagnosis, hippocampal volumes, WM ability as well as ADHD symptomatology, a K-means cluster analysis was performed with 245 participants: 111 TD, 58 ADHD-C, and 76 ADHD-I. The analysis included variables of brain morphology, behaviour, and cognition (Z-scored). The model included, left CA3 volume $(p=0.018)$, peer victimization score $(p<0.001), W M I(p<0.001)$, and the hyperactivity-impulsivity and inattention subscales from the SWAN (both $p<0.001$ ). Refer to Table 2 for a summary of the clusters. 
Table 2

Three-Cluster Model Participant Demographics.

\begin{tabular}{|lllll|}
\hline & Cluster $\mathbf{1}$ & Cluster 2 & Cluster 3 & P value \\
& $\mathbf{n = 4 1}$ & $\mathbf{n = 5 4}$ & $\mathbf{n = 1 5 0}$ & \\
\hline Left CA3 $\left(\mathrm{mm}^{3}\right)$, & 180.00 & 183.01 & 191.08 & 0.018 \\
Median [IQR] & {$[155.82-196.24]$} & {$[169.90-207.00]$} & {$[172.81-211.52]$} & $<0.001$ \\
WMI & 107.00 & 100.00 & 97.00 & $<0.001$ \\
Median [IQR] & {$[98.50-122.00]$} & {$[88.00-110.00]$} & {$[88.00-104.00]$} & $<0.001$ \\
Peer Victimization & 0.00 & 2.00 & 0.00 & $<0.001$ \\
Median [IQR] & {$[0.00-0.00]$} & {$[2.00-3.25]$} & {$[0.00-0.00]$} & \\
SWAN & -1.22 & 0.72 & 0.22 & \\
Hyperactivity-Impulsivity & {$[-2.78--0.56]$} & {$[0.22-1.44]$} & {$[0.00-0.78]$} & \\
Median [IQR] & -1.56 & 1.00 & 0.78 & \\
Inattention & {$[-2.22--0.78]$} & {$[0.22-1.67]$} & {$[0.11-1.44]$} & \\
Median [IQR] & & & & \\
\hline
\end{tabular}

Cluster 1 included a total of 41 participants and is characterized as children with small left CA3 volumes, high WMI scores, low peer victimization scores, and low hyperactivity-impulsivity and inattention symptomatology scores. This group is made up of 40 TD participants, and 1 participant from the ADHD-I group. All but 3 participants in this cluster scored 0.5 SD below the mean on the peer victimization scale. In this cluster $56 \%$ of the participants had left CA3 volumes that were below the average and $75 \%$ of the participants had higher average WMI. In regard to symptoms of hyperactivity-impulsivity and inattention, $39 \%$ of the participants in cluster 1 scored 1 SD below the mean and $34 \%$ scored below 2 SD below the mean.

Cluster 2 included a total of 54 participants. Participants in this group have average left CA3 volumes, average WMI scores, high peer victimization scores, and high hyperactivity-impulsivity and inattention symptomatology. This group is made up of 19 TD participants, 24 ADHD-C participants and 11 ADHD-I participants. All participants in this cluster were above average in peer victimization scores, with approximately $41 \%$ of the cluster at least 2 SD above the mean, and $56 \%$ above 1 SD. In this cluster $54 \%$ of participants had left CA3 volumes that were below average and $20 \%$ of participants had WMI at least 1 SD below the average. In regard to symptomatology, $20 \%$ of the participants had hyperactivity-impulsivity scores at least $1 \mathrm{SD}$ above the mean, and $28 \%$ had inattention scores at least 1 SD above the mean.

Cluster 3 included a total of 150 participants and can be described as participants with large left CA3 volumes, low WMI scores, low peer victimization scores, and average hyperactivity-impulsivity and 
inattention symptomatology. This cluster is made up of 52 TD participants, 34 ADHD-C participants, and 64 participants from the ADHD-I group. In cluster 3, approximately $79 \%$ of the participants had peer victimization scores that were 0.5 SD below the mean, A total of $60 \%$ of the participants had left CA3 volumes that were above the mean, with $21 \%$ being least 1 SD above the average. Approximately $30 \%$ of this cluster had WMI above the mean, and most participants in this cluster were above average in hyperactivity-impulsivity and inattention symptomatology, $57 \%$ and $60 \%$ respectively.

When examining cluster membership by diagnostic groups, we found $36 \%$ of the total TD sample $(n=$ 111) was grouped into cluster $1,17 \%$ were grouped into cluster 2 , and $47 \%$ were grouped into cluster 3 . Of the 58 total ADHD-C participants, $41 \%$ were grouped into cluster 2, and $59 \%$ were grouped into cluster 3 . Of the 76 total ADHD-I participants, $1 \%$ belonged to cluster 1, $14 \%$ were grouped into cluster 2 , and $84 \%$ were grouped into cluster 3 . Refer to Fig. 2 for a summary of the cluster analysis.

A one-way ANOVA was conducted to examine the differences between the clusters on all the variables. Significant between-group differences were found for all the variables: left CA3 volume $(F(2,242)=4.07$, $p=0.018)$, WMI $(F(2,242)=15.44, p<0.001)$, peer victimization $(F(2,242)=364.86, p<0.001)$, hyperactivity-impulsivity $(F(2,242)=103.63, p<0.001)$, and inattention $(F(2,242)=112.24, p<0.001)$. Post hoc multiple comparison using Bonferroni correction revealed the differences between the clusters. Significant differences were found between Cluster 1 and Cluster 2 on WMI, peer victimization, hyperactivity-impulsivity, and inattention (all $p<0.001$ ). Significant differences were found between Cluster 1 and 3 on Left CA3 volume $(p=0.015)$, WMI, hyperactivity-impulsivity, and inattention (all $p<$ 0.001). Significant differences were found between Cluster 2 and 3 on peer victimization $(p<0.001)$ and hyperactivity-impulsivity $(p=0.001)$.

\section{Discussion}

The presence of problem behaviours, levels of peer victimization, hippocampal subfield morphology, and working memory were assessed in a large heterogenous sample of children and adolescents further divided by diagnostic group: TD, ADHD-C and ADHD-I. We found that problem behaviours and levels of peer victimization differed between TD, ADHD-C, and ADHD-I groups. We also report that hippocampal subfield volumes predict the presence of peer victimization levels in children with ADHD and TD children. Lastly, hippocampal subfield volumes were associated with working memory ability in children with ADHD and TD children. Peer victimization was highest in children that displayed high levels of hyperactivity-impulsivity. Findings suggest that children with ADHD-C who display elevated levels of hyperactivity-impulsivity may be at greater risk for peer victimization.

\section{Problem Behaviours, Peer Victimization and Diagnostic Group}

As hypothesized, children in the ADHD diagnostic groups had elevated levels problem behaviours and peer victimization compared to TD children. Parents of children with ADHD-C reported higher levels of 
aggressive behaviour, rule breaking behaviour, social problems, and higher levels of experiencing peer victimization in their children compared to parental reports for TD and ADHD-I children. The ADHD-I children only differed from the TD children when comparing levels of aggressive behaviour; with significantly higher reported levels in the ADHD-I group. No significant differences were found in levels of withdrawal between any of the diagnostic groups.

Children and adolescents with ADHD are typically not well-liked by their peers and often experience social rejection and victimization ${ }^{5}$. Children with ADHD who display more severe levels of externalizing behaviours have been observed to be the most at-risk pediatric population to experience peer victimization $^{5,6}$. Investigation into how peer victimization differs amongst the diagnosis subtypes of $A D H D$ is sparse. Recent findings into the symptomatology of $A D H D$ in relation to peer victimization suggest that more severe symptoms of hyperactivity-impulsivity are associated with higher rates of peer victimization among children ${ }^{7}$. Not only are hyperactive-impulsive symptoms related to experiencing more victimization, it has also been demonstrated that individuals with these symptoms are also more likely to also be perpetrators of bullying themselves ${ }^{7}$. This notion is consistent with the findings from the present study. Although the peer victimization measure in the present study only accounted for experiencing victimization, the significantly elevated aggressive behaviour, rule breaking behaviour, and social problems scores in the ADHD-C group suggest that this group might also be perpetrators of victimization as well and might fit the profile of a bully victim ${ }^{7}$. As the ADHD-I group has levels of hyperactivity-impulsivity that are comparable to the TD group, it follows that they are victimized by their peers at rates similar to the TD group. Although the ADHD-I children also had significantly elevated aggression scores compared to TD children, the lack of hyperactivity-impulsivity symptoms combined with low rates of peer victimization suggest that this group might fit into a different category than the ADHD-C group; that is, one of aggressive children and adolescents that are accepted by their peers ${ }^{34}$.

Experiencing peer victimization may be traumatizing, with a tremendous impact on later psychological development ${ }^{35}$. It is crucial that future research focuses on examining the impact of different types of victimization on child and adolescent psychological health. Determining how different types of victimization effect children can inform intervention strategies and can be useful for informing schoolbased interventions.

\section{Hippocampal Subfield Volume, Problem Behaviours and Peer Victimization}

In the current study, contrary to our hypothesis, larger left CA3 volumes were associated with higher parent-reported rates of peer victimization in children with ADHD-C, ADHD-I and TD children. The CA3 region is a particularly stress-sensitive area and has previously been associated with early life adversity ${ }^{8,36}$. Typically, smaller hippocampal volumes have been associated with a greater incidence of trauma and adversity in TD children ${ }^{16,17,36}$. However, this association has been found to be different in children with mental health and neurodevelopmental disorders ${ }^{18,21,37}$. Some models suggest that 
chronically elevated levels of stress during childhood may cause reduced stress responsivity which in turn may mitigate the neurotoxic effects GCs typically have in atrophying the neurons of the hippocampus ${ }^{17,18,19}$. This cascade of events results in larger hippocampal volumes, as found in our study.

Research conducted with pediatric post-traumatic stress disorder (PTSD) populations has provided evidence for larger hippocampal volumes when compared to matched non-traumatized groups, suggesting that anxiety and stress may be associated with increased growth of the hippocampus ${ }^{21}$. A recent study found larger subiculum, presubiculum, and CA1 volumes to be associated with childhood trauma in bipolar children ${ }^{18}$. Children with ASD have also been found to have enlarged hippocampal volumes compared to TD children ${ }^{37}$ and larger hippocampal volumes in children with ADHD have also been observed ${ }^{12}$. Plessen ${ }^{12}$ et al. found hippocampal volumes to be inversely related to symptom severity in ADHD-C, whereas Tupler \& De Bellis ${ }^{21}$ found larger hippocampal volumes to be associated with increased externalizing symptoms.

Future research in this area should focus on examining if and how the relationship between hippocampal volume and peer victimization differs according to gender and age. To maintain the large sample size in the current study we were not able to split our population in these ways; however, these factors were used as covariates. Age was not a significant covariate in the model relating hippocampal volumes and peer victimization. In addition, future research is warranted in elucidating the relationship between ADHD subtype, symptomatology and peer victimization in relation to hippocampal volume, specifically in including the ADHD-H subtype.

\section{Hippocampal Subfield Volume and Working Memory}

The ADHD-I children had significantly lower scores on the WMI when compared to TD children. The ADHD-C group did not significantly differ from the ADHD-I group nor the TD group on WMI scores. Working memory deficits are often seen in children with an ADHD diagnosis ${ }^{26,38}$. However, there is a lack of consensus on how the different ADHD subtypes differ in respect to working memory ability, and cognitive ability in general. Some studies report no differences between high and low inattention symptoms and working memory scores ${ }^{39}$. Other studies have found inattention to be a significant predictor of working memory ability in adolescents with $A D H D^{40}$. As children with ADHD-C also have clinically elevated symptoms of inattention, it is unclear why the ADHD-C children did not display WM deficits when compared to the TD children in our study.

Larger left CA3 volumes and smaller left CA4 volumes were significantly predictive of higher WMI scores in all of the diagnostic groups. CA3 and CA4 are both key hippocampal subfields that are involved in $W^{41}$. Larger volumes predict better cognitive function in adult samples ${ }^{24,25,31}$. Yet, findings in children and adolescents provide inconsistent results to support this view. In a meta-analysis by Van Petten ${ }^{24}$ multiple studies examining hippocampal volume and memory performance in child and adolescent populations found negative correlations between volume and memory abilities. This suggests that the 
positive relationship between cognitive ability and hippocampal brain volume may increase with age ${ }^{24}$. A recent study found that as children age, smaller hippocampal volumes are associated with superior memory abilities ${ }^{42,43}$. Previous studies examined whole hippocampal volumes. Future studies should investigate the relationship between hippocampal subfield volumes and working memory ability in ADHD populations.

\section{ADHD Subtypes, Hippocampal volumes, Working memory and Peer Victimization}

A three-cluster K-means model was used to characterize the present study population. Cluster 1 included 40 TD children and 1 child with ADHD-I who have low levels of inattentive and hyperactive-impulsive symptomatology, good working memory ability, and low peer victimization. Cluster 2 included 19 TD, 24 ADHD-C and 11 ADHD-I children who have significantly elevated levels of peer victimization and hyperactivity-impulsivity when compared to the two other clusters. Cluster 3 included 52 TD, 34 ADHD-C and 64 ADHD-I children who have significantly enlarged left CA3 volumes and significantly higher hyperactivity-impulsivity and WMIs compared to children in Cluster 1.

The results of the cluster analysis suggest that ADHD symptomatology, especially hyperactivityimpulsivity, is related to increased levels of peer victimization. Our model also suggests that larger left CA3 volumes are associated with ADHD symptomatology. In both Clusters 2 and 3 , symptoms hyperactivity-impulsivity and inattention are significantly elevated and, in both groups, left CA3 volumes are enlarged compared to Cluster 1 , although only significantly enlarged in Cluster 3 . Similarities can be seen between our model and the inverse correlation Plessen ${ }^{12}$ et al. observed between CA3 volume and symptoms of inattention in children with ADHD. In our model children in Cluster 3 have the most prominent enlargement of left CA3 volumes, but milder symptoms of inattention than children in Cluster 2. This suggests the possibility that enlarged left CA3 volumes are representative of a compensatory mechanism by which the hippocampus hypertrophies in response to the presence of ADHD symptoms and results in less severe symptomatology ${ }^{12}$. The relationship between symptoms of inattention in children with ADHD and hippocampal volume, specifically in the CA3 subfield should be further explored. Our results suggest that deep phenotyping of brain morphology, cognition, and behaviour can identify subtle differences in ADHD subtypes.

A limitation of the current study is our use of a parent-report questionnaire, the CBCL, as a measure of problem behaviour and peer victimization. Children have many experiences without their parents present, such as in school, recreational activities, etc., thus child reports may be able to provide additional information that cannot be captured by parent-report alone.

Youth Self Report data was available however not enough children and adolescents completed this questionnaire, which drastically reduced the sample size and power of analyses. Another limitation of the current study was the absence of the ADHD hyperactive/impulsive subtype (ADHD-H). We only had access to data for 15 participants diagnosed with ADHD-H (16.5\% of the total ADHD sample), which was 
not a large enough sample for valid analyses. In children and adolescents ranging from 3 to 18 years of age, ADHD-I is the most common ADHD subtype with a prevalence of between 2.2-5.7\%, the prevalence of ADHD-C ranges from 1.1-2.4\%, and the prevalence of ADHD-H ranges from 1.1-4.9\% ${ }^{49}$. As children age, an ADHD-H diagnosis becomes less common and could be the reason we had access to the data of very few children with this subtype. Another limitation is that different scanners, with different strengths were used at the various MRI sites. However, a majority of the participants were scanned at CBIC and RU, which both had $3 T$ scanners. The medication status of the children and adolescents in the study sample are unknown and this is a potential confound for behavioural reports, hippocampal volumes and WM performance.

\section{Declarations}

\section{Author Contributions}

A.P., E.G.D., and D.S. were involved in study design and conception. A.P. conducted data analysis, interpreted the results, and wrote the manuscript text. S.C. pre-processed the Magnetic Resonance Imaging data. A.P, E.G.D., and D.S reviewed the manuscript.

\section{Corresponding Author}

Correspondence to Emma G. Duerden.

\section{Competing Interests}

The authors declare no competing interests.

\section{Data availability statement}

The datasets generated and/or analysed during the current study are available in the Child Mind Institute Healthy Brain Network repository, http://fcon_1000.projects.nitrc.org/indi/cmi_healthy_brain_network/

\section{Funding}

The work presented here was primarily supported by gifts to the Child Mind Institute from Phyllis Green, Randolph Cowen, and Joseph Healey, as well as NIMH awards to Dr. Milham (U01MH099059, R01MH091864). This research was also supported by the Intramural Research Program of the NIMH (Merikangas; grant number ZIAMH002953). The funders for this project were not involved in any part of the experimental design, analysis and interpretation of data, or manuscript preparation and submission. MPM is the Phyllis Green and Randolph Cowen Scholar; Joe Healey provides philanthropic gifts to the Center for the Developing Brain.

\section{References}


1. American Psychiatric Association. Diagnostic and statistical manual of mental disorders (DSM-5®) (American Psychiatric Pub, 2013).

2. Polanczyk, G., De Lima, M. S., Horta, B. L., Biederman, J. \& Rohde, L. A. The worldwide prevalence of ADHD: a systematic review and metaregression analysis. Am. J. Psychiatry. 164, 942-948 (2007).

3. Gadow, K. D. et al. Comparison of ADHD symptom subtypes as source-specific syndromes. J. of Child Psycho. and Psychiatry. 45, 1135-1149 (2004).

4. Frankel, F. \& Feinberg, D. (2002). Social problems associated with ADHD vs. ODD in children referred for friendship problems. Child Psychiatry Hum. Dev. 33, 125-146 (2002).

5. Humphrey, J. L., Storch, E. A. \& Geffken, G. R. Peer victimization in children with attention-deficit hyperactivity disorder. J. Child Health Care. 11, 248-260 (2007).

6. Blake, J. J. et al. Predictors of bully victimization in students with disabilities: A longitudinal examination using a national data set. J. Disabil. Policy Stud. 26, 199-208 (2016).

7. Winters, R. R., Blake, J. J. \& Chen, S. Bully victimization among children with attentiondeficit/hyperactivity disorder: a longitudinal examination of behavioral phenotypes. J. Emot. Behav. Disord. 28, 80-91 (2020).

8. Teicher, M. H. et al. Differential effects of childhood neglect and abuse during sensitive exposure periods on male and female hippocampus. Neuroimage. 169, 443-452 (2018).

9. Quinlan, E. B. et al. Peer victimization and its impact on adolescent brain development and psychopathology. Mol. Psychiatry. 25, 3066-3076 (2018).

10. Shaw, P. et al. Attention-deficit/hyperactivity disorder is characterized by a delay in cortical maturation. Proc. Natl. Acad. Sci. U.S.A. 104, 19649-19654(2007).

11. Harms, M. P., Wang, L., Csernansky, J. G. \& Barch, D. M. Structure-function relationship of working memory activity with hippocampal and prefrontal cortex volumes. Brain Sruct. Funct. 218, 173-186 (2013).

12. Plessen, K. J. et al. Hippocampus and amygdala morphology in attention-deficit/hyperactivity disorder. Arch. Gen. Psychiatry. 63, 795-807 (2006).

13. Hoogman, M. et al. Subcortical brain volume differences in participants with attention deficit hyperactivity disorder in children and adults: a cross-sectional mega-analysis. Lancet Psychiat. 4, 310-319 (2017).

14. Posner, J. et al. A multimodal MRI study of the hippocampus in medication-naive children with ADHD: what connects ADHD and depression? Psychatry Res. Neuroimaging. 224, 112-118 (2014).

15. Perlov, E. et al. Hippocampus and amygdala morphology in adults with attention-deficit hyperactivity disorder. J. Psychatry Neurosci.: 33, 509 (2008).

16. Dahmen, B. et al. Effects of early-life adversity on hippocampal structures and associated HPA axis functions. Dev. Neurosci. 40, 13-22 (2018).

17. Sapolsky, R. M. Glucocorticoids and hippocampal atrophy in neuropsychiatric disorders. Arch. Gen. Psychiatry. 57, 925-935 (2000). 
18. Janiri, D. et al. Hippocampal subfield volumes and childhood trauma in bipolar disorders. J. Affect. Disord. 253, 35-43 (2019).

19. Liberzon, I. \& Abelson, J. L. Context processing and the neurobiology of post-traumatic stress disorder. Neuron. 92, 14-30 (2016).

20. Humphreys, K. L. et al. Evidence for a sensitive period in the effects of early life stress on hippocampal volume. Dev. Sci. 22, e12775 (2019).

21. Tupler, L. A. \& De Bellis, M. D. Segmented hippocampal volume in children and adolescents with posttraumatic stress disorder. Biol. Psychiatry. 59, 523-529 (2006).

22. Lee, S. W. et al. Hippocampal subfields Vol. reduction in high schoolers with previous verbal abuse experiences. Clin. Psychopharmacol. Neurosci. 16, 46-56 (2018).

23. McDaniel, M. A. Big-brained people are smarter: A meta-analysis of the relationship between in vivo brain volume and intelligence. Intelligence. 33, 337-346 (2005).

24. Van Petten, C. Relationship between hippocampal volume and memory ability in healthy individuals across the lifespan: review and meta-analysis. Neuropsychologia. 42, 1394-1413 (2004).

25. Beauchamp, M. H. et al. Preterm infant hippocampal volumes correlate with later working memory deficits. Brain. 131, 2986-2994 (2008).

26. Martinussen, R., Hayden, J., Hogg-Johnson, S. \& Tannock, R. A meta-analysis of working memory impairments in children with attention-deficit/hyperactivity disorder. J. Am. Acad. Child Psy. 44, 377384 (2005).

27. Alexander, L. M. et al. An open resource for transdiagnostic research in pediatric mental health and learning disorders. Sci Data. 4, 1-26 (2017).

28. Kaufman, J. et al. Schedule for affective disorders and schizophrenia for school-age children-resent and lifetime version (K-SADS-PL): Initial reliability and validity data. J. Am. Acad. Child Psy. 36, 980988 (1997).

29. Barratt, W. The Barratt simplified measure of social status (BSMSS)(Indiana State University, 2006).

30. Achenbach, T. M. Integrative Guide to the 1991 CBCL/4-18, YSR, and TRF Profiles(University of Vermont Department of Psychology, 1991).

31. McCloskey, L. A. \& Stuewig, J. The quality of peer relationships among children exposed to family violence. Dev. Psychopathol. 13, 83-96 (2001).

32. Wechsler, D. (2014). The Wechsler intelligence scale for children-fifth edition (The Psychological Corporation, 2014).

33. Iglesias, J. E. et al. A computational atlas of the hippocampal formation using ex vivo, ultra-high resolution MRI: application to adaptive segmentation of in vivo MRI. Neuroimage. 115, 117-137 (2015).

34. Farmer, T. et al. Peer relations of bullies, bully-victims, and victims: The two social worlds of bullying in second-grade classrooms. Elem. Sch. J. 110, 364-392 (2010). 
35. Wolke, D., Copeland, W. E., Angold, A. \& Costello, E. J. Impact of bullying in childhood on adult health, wealth, crime, and social outcomes. Psychol. Sci. 24, 1958-1970 (2013).

36. Teicher, M. H., Anderson, C. M. \& Polcari, A. Childhood maltreatment is associated with reduced volume in the hippocampal subfields CA3, dentate gyrus, and subiculum. Proc. Natl. Acad. Sci. U.S.A. 109, E563-E572(2012).

37. Groen, W., Teluij, M., Buitelaar, J. \& Tendolkar, I. Amygdala and hippocampus enlargement during adolescence in autism. J. Am. Acad. Child Psy. 49, 552-560 (2010).

38. Ramos, A. A., Hamdan, A. C. \& Machado, L. A meta-analysis on verbal working memory in children and adolescents with ADHD. Clin. Neuropsychol. 34, 873-898 (2020).

39. Jonkman, L. M., Markus, C. R., Franklin, M. S. \& van Dalfsen, J. H. Mind wandering during attention performance: Effects of ADHD-inattention symptomatology, negative mood, ruminative response style and working memory capacity. PLoS One. 12, e0181213 (2017).

40. Rogers, M., Hwang, H., Toplak, M., Weiss, M. \& Tannock, R. Inattention, working memory, and academic achievement in adolescents referred for attention deficit/hyperactivity disorder (ADHD). Child Neuropsychol. 17, 444-458 (2011).

41. Voineskos, A. N. et al. Hippocampal (subfield) volume and shape in relation to cognitive performance across the adult lifespan. Hum. Brain. Mapp. 36, 3020-3037 (2015).

42. Keresztes, A. et al. Hair cortisol concentrations are associated with hippocampal subregional volumes in children. Sci. Rep. 10, 1-12 (2020).

43. Riggins, T. et al. Protracted hippocampal development is associated with age-related improvements in memory during early childhood. Neuroimage. 174, 127-137 (2018).

44. Willcutt, E. G. The prevalence of DSM-IV attention-deficit/hyperactivity disorder: a meta-analytic review. Neurotherapeutics. 9, 490-499 (2012).

\section{Figures}




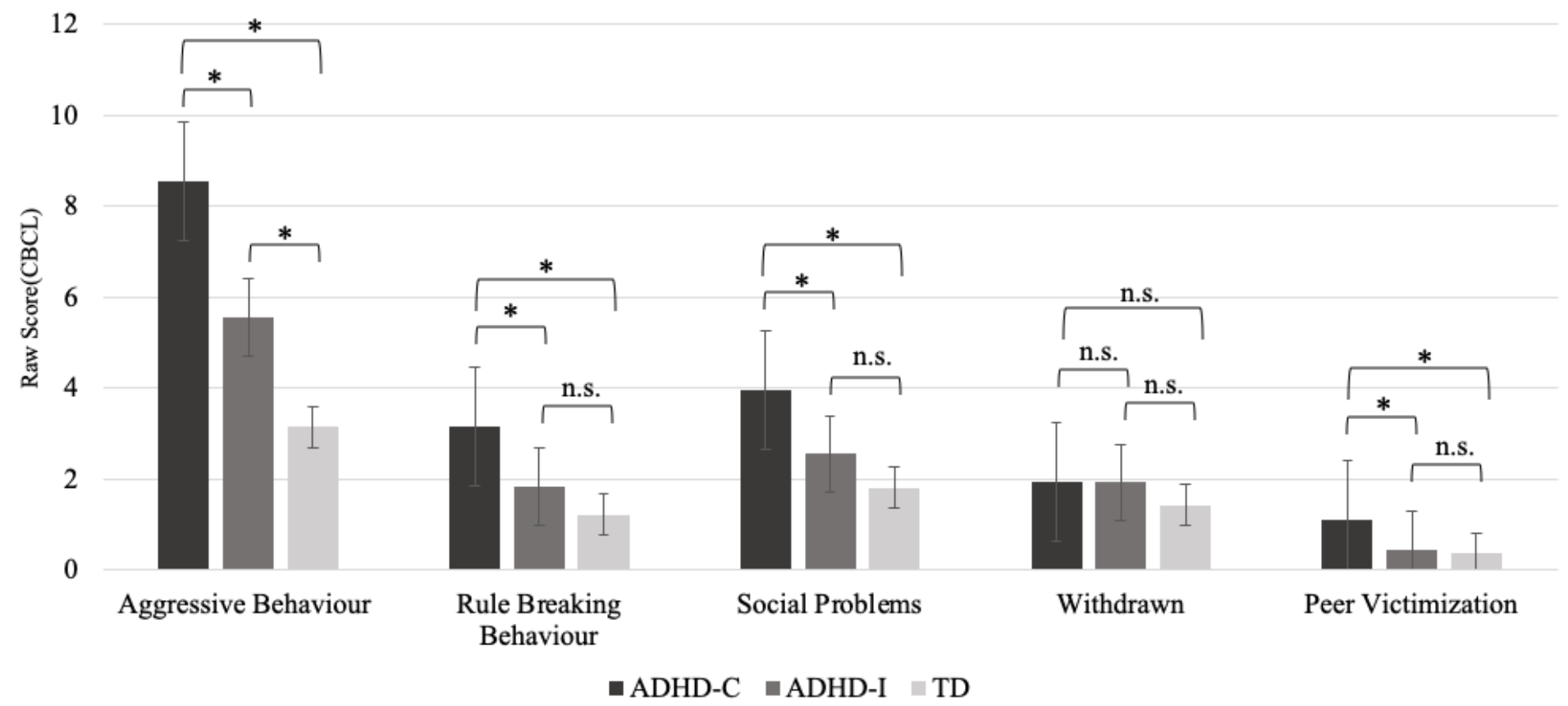

\section{Figure 1}

Problem Behaviours and Peer Victimization in Diagnostic Groups. CBCL subscale scores for children and adolescents from the ADHD-C, ADHD-I, and TD groups. Children and adolescents from the ADHD-C group had significantly higher Aggressive Behaviour, Rule Breaking Behaviour, Social Problems, and Peer Victimization scores compared to the TD group. The ADHD-C group also had significantly higher Aggressive Behaviour, Rule Breaking Behaviour, Social Problems and Peer Victimization scores compared to the ADHD-I group. Children and adolescents in the ADHD-I group had significantly higher Aggressive Behaviour scores than the TD group. None of the groups significantly differed on the Withdrawn scores. Scores represent the estimated marginal means, adjusted for age, sex, study site, and SES. P values are Bonferroni corrected (pairwise) for multiple comparisons. Error bars reflect standard error. ${ }^{*} p<0.001$ 

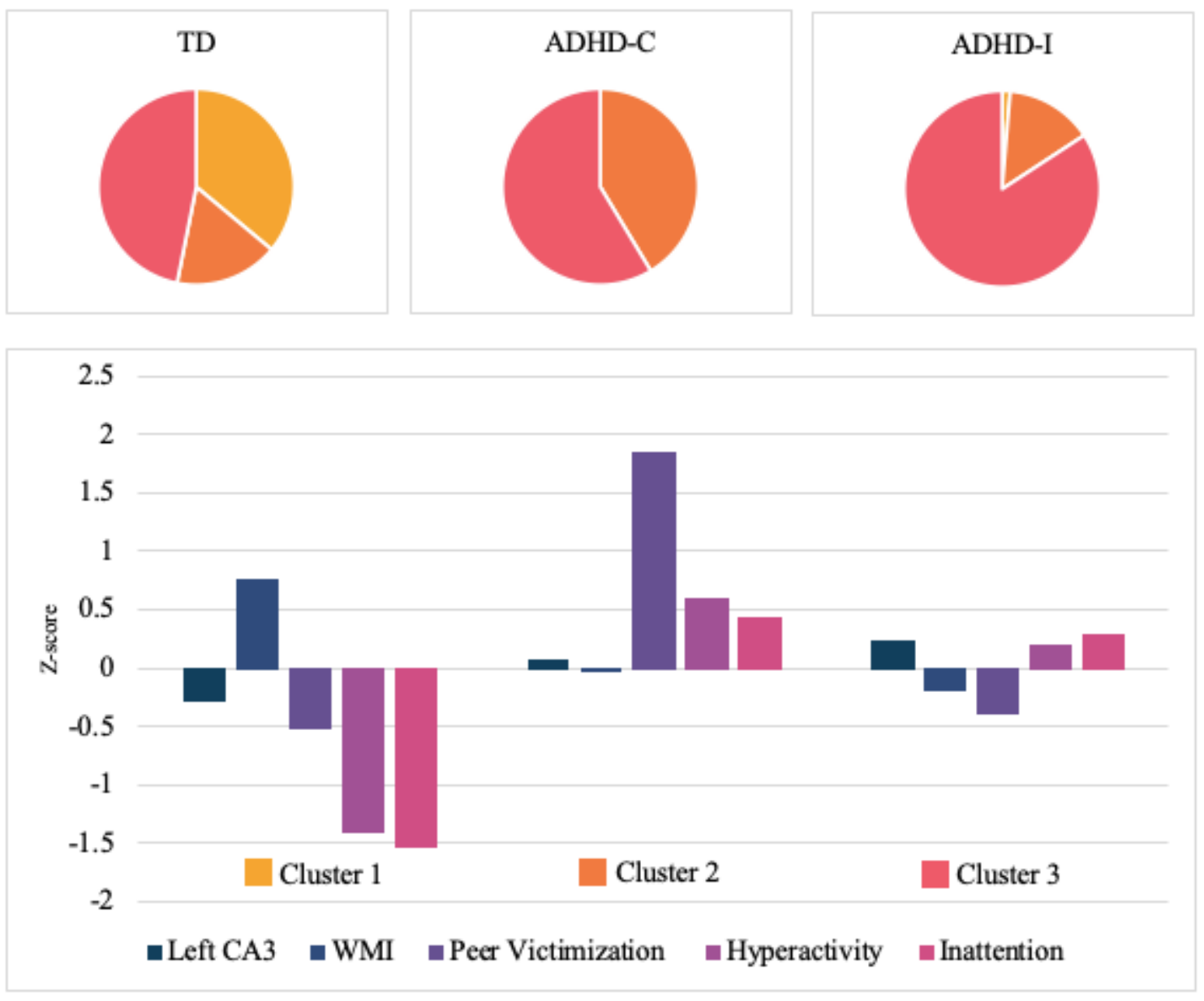

Figure 2

K-means Clustering based on Peer Victimization, Left CA3, WMI, Hyperactivity-Impulsivity and Inattention. The three-cluster model is depicted above. Cluster 1 is characterized by small left CA3 volume, high WMI, low peer victimization, and low hyperactivity-impulsivity and inattention. Cluster 1 is almost entirely made up of TD participants. Cluster 2 is characterized as average left CA3 volumes, average WMI, high peer victimization, and high hyperactivity-impulsivity and inattention. Of the participants in cluster $2,44 \%$ are from the ADHD-C group. Cluster 3 is characterized as large left CA3 volume, low WMI, low peer victimization, and average hyperactivity-impulsivity and inattention. Cluster 3 is approximately. $43 \%$ ADHD-I participants, 35\% TD participants, and 22\% ADHD-C participants. 